\title{
Polar bodies are efficient donors for reconstruction of human embryos for potential mitochondrial replacement therapy
}

Cell Research (2017) 27:1069-1072. doi:10.1038/cr.2017.67; published online 12 May 2017

\section{Dear Editor,}

In mice, the first $\left(1^{\text {st }}\right)$ and second $\left(2^{\text {nd }}\right)$ polar bodies (PBs) can be used in place of the female genome of oocytes or zygotes and efficiently support the generation of embryonic stem cells (ESCs) and normal offspring [13]. These results indicate that both $1^{\text {st }}$ and $2^{\text {nd }} P B s$ can be used as nuclear donors for mitochondrial replacement therapy (MRT) [4], which is an effective approach for preventing the transmission of disease-causing mutant mitochondria from mother to children [5]. Most recently, human $1^{\text {st }}$ polar body (PB1) has been successfully used to replace the maternal genome to generate functional oocytes, demonstrated by the generation of blastocysts after fertilization [6]. However, whether human $2^{\text {nd }}$ polar body (PB2) can be used as the nuclear donor for embryo reconstruction remains unknown. In this study, we establish protocols for the reconstruction of human oocytes or zygotes using $1^{\text {st }}$ or $2^{\text {nd }} P B s$ as donors under conditions without cytoskeleton disruptors. Normal human ESCs can be efficiently generated from reconstructed embryos by PB transfer (PBT). Importantly, mitochondrial DNA (mtDNA) carryover remains at low levels during subsequent ESC culture and differentiation.

To reconstruct human oocytes with PB1, we collected MI oocytes donated by patients who were taking in-vitro fertilization (IVF) treatments. In vitro maturation of MI oocytes was performed to obtain matured oocytes (MII oocytes) with the distinct PB1. MII oocytes were randomly divided into two groups as donors or recipients. PB1s were recovered using a pipette with an inner diameter of $15 \mu \mathrm{m}$ and briefly incubated with Sendai virus extract [7], followed by injection into the perivitelline space of the recipient oocytes at the opposite location of their PB1. Membrane fusion between the injected PB1 and the recipient oocyte occurred within $0.5 \mathrm{~h}$ and sperm was injected into the fused oocytes in $0.5 \mathrm{~h}$. The female pre-pronucleus (PPN) together with the extruding PB2 of the recipient oocyte, 3.5-4 $\mathrm{h}$ after sperm injection, could be distinguished according to its location close to PB1 of the recipient, and thus could be easily removed under the condition without Cytokalasin B (CB) [8], generating PB1-transferred embryos (PB1T embryos) (Figures 1A, $1 \mathrm{~B}$ and Supplementary information, Movie S1).

A total of 75 PB1T embryos were successfully generated and cultured in vitro for 5 or 6 days. 19 good-quality blastocysts (4BC or better by Gardner's criteria) were obtained and cryopreserved until further analyses (Supplementary information, Table S1A and Figure S1A). 17 of them were successfully thawed. To test the genome origin of PB1T blastocysts, DNA samples were extracted from trophoblast cells dissociated by trophectoderm (TE) biopsy of 16 embryos and used for short tandem repeat (STR) genotyping. As expected, all of them showed precise match with the corresponding $\mathrm{PB} 1$ donors, demonstrating a high success rate of generation of PB1T embryos by our strategy (Supplementary information, Table S1B and S1C). We next analyzed the karyotype of PB1T embryos using the same DNA samples for preimplantation genetic screening (PGS). The results showed that 10 blastocysts were euploid. However, the rest 6 embryos sustained aneuploidy (Supplementary information, Table S1C), consistent with previous observations of generation of aneuploid embryos by IVF centers [9, 10] and recent observations in human PB1T experiments [6]. Moreover, droplet digital PCR (ddPCR) [11] showed that the 10 blastocysts with normal karyotype displayed an average mtDNA carryover level of $0.26 \%(0.07 \%$ $0.50 \%$ ) (Supplementary information, Figure S1B). Taken together, these results demonstrate that PB1 can be used as nuclear donors for reconstruction of human oocytes.

We next tested whether human PB2 could also be used as donors for reconstruction of human embryos. To this end, MII oocytes that matured from donated MI oocytes were adopted for intracytoplasmic sperm injection (ICSI) followed by removal of PB1s. To prepare recipients, the female PPN with the extruding PB2 was removed 3.5-4 $\mathrm{h}$ after ICSI under the condition without 
CB according to a protocol reported previously [8]. To prepare donors, $\mathrm{PB} 2$ was collected using a pipette with an inner diameter of $15 \mu \mathrm{m} \mathrm{6-7} \mathrm{h}$ after ICSI when PB2 was completely separated from the female pronucleus. PB2s, after a brief incubation with Sendai virus extract [7], were injected into the perivitelline space of the recipients. The fused embryos, so-called PB2-transferred (PB2T) embryos, with two pronuclei (2PN) observed on the following day were cultured in vitro to the blastocyst stage (Figure 1C-1E and Supplementary information, Movie S2). As shown in Supplementary information, Table S1A, a total of 14 high-quality PB2T blastocysts were generated from 51 PB2T embryos, at a comparable efficiency to PB1T embryos and control ICSI embryos. STR analysis of TE biopsy samples confirmed the PB2 origin of PB2T blastocysts (Supplementary information, Table S1B and S1C). PGS analysis indicated that 9 blastocysts were euploid (Supplementary information, Table S1C). This euploidy rate is similar to that observed in PB1T blastocysts and previous studies [6,9]. Moreover, the 9 blastocysts with normal karyotype showed an average mtDNA carryover level of $0.37 \%(0.06 \%-0.70 \%)$ (Figures 1F). Taken together, these data indicate that our PBT protocols do not affect pre-implantation development of reconstructed embryos.

Having demonstrated the successful reconstruction of human embryos using both PB1 and PB2 as donors to replace the maternal genome, we next tested mtDNA carryover levels in ESCs derived from our PBT embryos. To this end, a total of 5 and 7 ESC lines were obtained from PB1T and PB2T blastocysts with normal karyotypes, respectively (Supplementary information, Figure S2A and Table S1C). G-banding analysis indicated that all tested cells exhibited normal karyotypes (Figure $1 \mathrm{G}$ and Supplementary information, Figure S2B). These cells displayed typical human ESC characteristics, including maintenance of colony morphology, expression of human ESC markers and exhibition of typical human ESC gene expression and methylation profiles (Figure $1 \mathrm{H}$ and Supplementary information, Figure S2C-S2E). Moreover, PBT ESCs could differentiate into cells of the three germ layers in vitro and in vivo (Supplementary information, Figure S2F and S2G). We then analyzed the levels of mtDNA carryover at different passages during ESC in vitro proliferation by collecting DNA samples every five passages. The ddRCR results from $3 \mathrm{~PB} 1 \mathrm{~T}$ and $6 \mathrm{~PB} 2 \mathrm{~T}$ ESC lines showed that the ratio of mtDNA carryover was relatively stable and maintained at low levels during ESC proliferation (Figure 1I and Supplementary information, Table S1C). Finally, DNA samples of embryoid bodies (EBs) and teratomas were prepared for ddPCR analysis. The results showed that mtDNA carryover was stably maintained at very low levels during ESC differentiation in vitro and in vivo (Figure 1I and Supplementary information, Table S1C). Taken together, these results demonstrate that our PBT procedures can efficiently produce reconstructed blastocysts and ESCs with very low levels of mtDNA carryover.

The developmental potential of zygotes with PB1 and PB2 as donors for nuclear transfer has been investigated decades ago $[2,3]$ and recently their application in MRT has been comprehensively tested in mice [4]. The PBs have smaller size compared with chromosome-spindle complexes and pronuclei, and thus have very few mitochondria attached $[4,12]$, making them suitable donors for MRT. In this study, we extend mouse discoveries to human by showing that both human PB1 and PB2 can be efficiently adopted as donors for reconstruction of human embryos, from which ESCs can be generated. Meanwhile, we show that the reconstructed blastocysts and the derived ESCs carry very low levels of mtDNAs originated from PB donors. Importantly, mtDNA carryover was maintained at low levels during long-term in vitro proliferation and differentiation of ESCs, suggesting that $\mathrm{PBT}$ can be a promising approach to treat mtDNA-related diseases. Our approach has a number of advantages over existing technologies for MRT. First, the membrane of PBs can protect the female genome from damages introduced by procedures of nuclear isolation and transfer. Second, our protocols allow the removal of PPN without using cytoskeleton disruptors. Third, it is easier to distinguish female and male PPNs based on the close proximity of female PPN to the extruding PB2 compared to current MRT protocols, in which a female pronucleus is recognized according to its smaller size or a location close to PB2. During the preparation of this manuscript, an independent study reported the generation of reconstructed human blastocysts using PB1 as donors [6]. Here, besides the PB1T protocol, we also established a PB2T protocol and evaluated the mtDNA carryover rates in both $\mathrm{PB} 1 \mathrm{~T}$ and $\mathrm{PB} 2 \mathrm{~T}$ strategies. The differences between PB1 and PB2 transfer have been summarized in Supplementary information, Data S1A. In summary, our data indicate that it is feasible to use PB1 and PB2 as nuclear donors for reconstruction of human embryos. These methods represent a potential strategy for preventing mtDNA disease transmission in human.

\section{Acknowledgments}

The authors acknowledge the Institutional Review Board of Reproductive Medicine, Shandong University for providing oversight and guidance. We thank all participants for their support in providing the gametes. We thank Y Chen and X Ding from the Stem Cell Core Facility at SIBCB for support with cell cultures, 
A

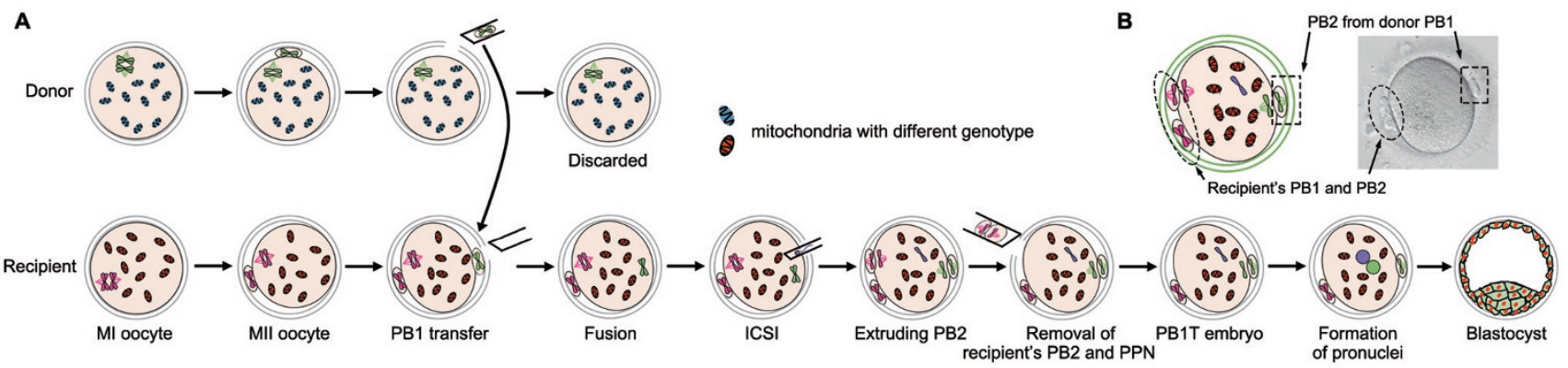

$C$
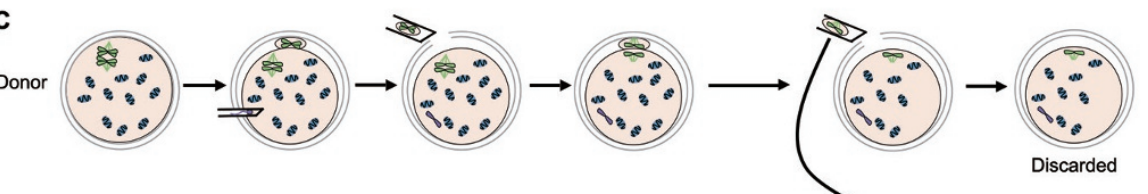

D Removal of recipient's PB2 and PPN Removi
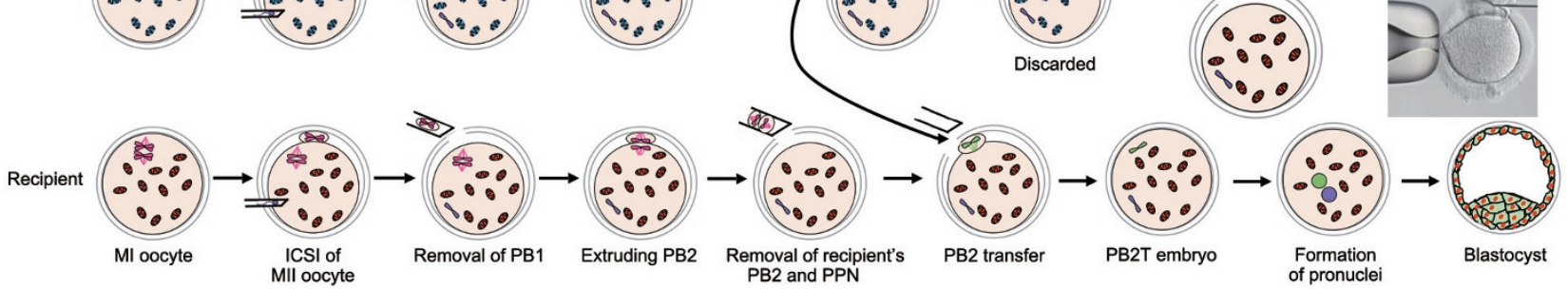

E

$\mathbf{F}$

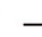

\begin{tabular}{cc}
\hline No. & mtDNA carryover \\
\hline PB2T-1 & $0.22 \%$ \\
PB2T-4 & $0.54 \%$ \\
PB2T-7 & $0.67 \%$ \\
PB2T-8 & $0.65 \%$ \\
PB2T-9 & $0.21 \%$ \\
PB2T-10 & $0.06 \%$ \\
PB2T-11 & $0.13 \%$ \\
PB2T-12 & $0.70 \%$ \\
PB2T-14 & $0.13 \%$ \\
Avg & $0.37 \%$
\end{tabular}

G

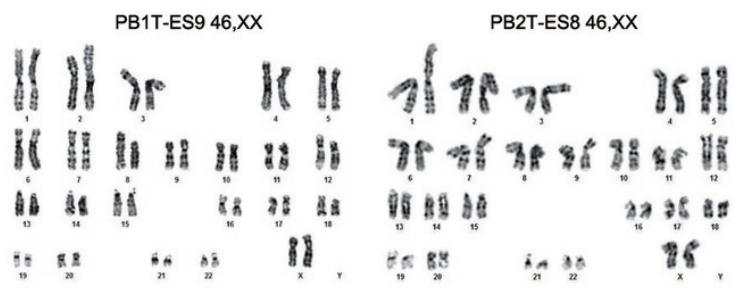

H

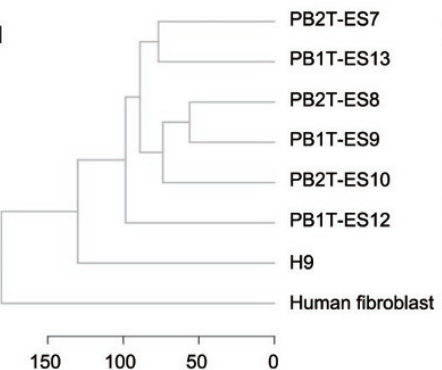

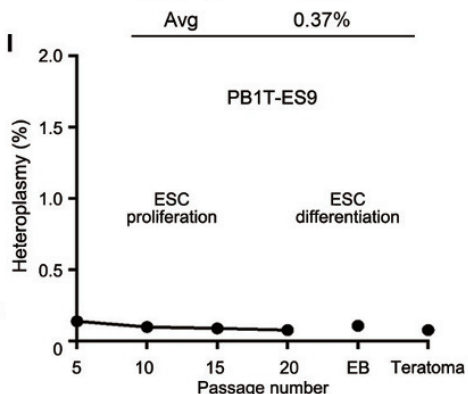

Figure 1 Human PB1 and PB2 can be used as nuclear donors for reconstruction of human embryos. (A) Schematic diagram of PB1T. (B) A representative image of the reconstructed embryo carrying three PBs, i.e., recipient's PB1 and PB2 and PB2 derived from donor PB1. (C) Schematic diagram of PB2T. (D) A representative image of removal of recipient's PPN and PB2. (E) Development of PB2T embryos. i, reconstructed embryo; ii, formation of pronuclei; iii, 2-cell embryo; iv, 4-cell embryo; v, 8-cell embryo; vi, blastocyst. (F) MtDNA carryover rates in PB2T blastocysts with normal karyotype detected by ddPCR analysis. (G) Representative images of G-banding analysis of ESCs derived from PB1T and PB2T blastocysts. (H) Gene expression profiles of human ESCs derived from PB1T and PB2T blastocysts were analyzed by RNA-seq. Gene expression profiles were clustered using all expressed genes. (I) MtDNA carryover analysis during ESC proliferation and differentiation.

S Huang, $\mathrm{P} \mathrm{Xu}$ and $\mathrm{Y}$ Peng from Shandong University for support with ddPCR and STR analysis, and R Hou and H Xiao from Shanghai Biochip Company for sequencing data analysis. This study was supported by the National Key Research and Development Program of China (2016YFC1000600), the Ministry of Sci- ence and Technology of China (2014CB964803, 2015AA020307 and 2013CB967103), the National Natural Science Foundation of China $(81430029,81490743,81622021,81601256,31371453$, 31571548,31530048 and 81672117), the Chinese Academy of Sciences (XDB19010204), the Postdoctoral Innovation Support 
Program (BX201600108) and the Shanghai Municipal Commission for Science and Technology (16JC1420500).

\section{Keliang Wu ${ }^{1,2,3, *}$, Cuiqing Zhong ${ }^{1,2,3,4,5,6, *}$, Tailai Chen ${ }^{1,2,3, *}$, Xiaoyu Zhang ${ }^{6}$, Wenrong Tao ${ }^{1,2,3}$, Jingye Zhang ${ }^{1,2,3}$, Hongchang $\mathrm{Li}^{1,2,3}$, Han Zhao ${ }^{1,2,3}$, Jinsong Li ${ }^{6,7}, \mathrm{Zi}$-Jiang Chen ${ }^{1,2,3,4,5}$}

${ }^{\text {I}}$ Center for Reproductive Medicine, Shandong Provincial Hospital Affiliated to Shandong University, Jinan, Shandong 250001, China; ${ }^{2}$ National Research Center for Assisted Reproductive Technology and Reproductive Genetics, Jinan, Shandong 250001, China; ${ }^{3}$ The Key laboratory for Reproductive Endocrinology of Ministry of Education, Jinan, Shandong 250001, China; ${ }^{4}$ Center for Reproductive Medicine, Ren Ji Hospital, School of Medicine, Shanghai Jiao Tong University, Shanghai 200135, China; ${ }^{5}$ Shanghai Key Laboratory for Assisted Reproduction and Reproductive Genetics, Shanghai 200135, China; ${ }^{6}$ State Key Laboratory of Cell Biology, Shanghai Key Laboratory of Molecular Andrology, CAS Center for Excellence in Molecular Cell Science, Shanghai Institute of Biochemistry and Cell Biology, Chinese Academy of Sciences \& University of Chinese Academy of Sciences, 320 Yueyang Road, Shanghai 200031, China; ${ }^{7}$ School of Life Science and Technology, ShanghaiTech University, 100 Haike Road, Shanghai 201210, China

*These three authors contributed equally to this work.

Correspondence: Zi-Jiang Chen ${ }^{\mathrm{a}}$, Jinsong $\mathrm{Li}^{\mathrm{b}}$, Han $\mathrm{Zhao}^{\mathrm{c}}$

${ }^{a}$ E-mail: chenzijiang@hotmail.com
bE-mail: jsli@sibcb.ac.cn

${ }^{c}$ E-mail: hanzh80@yahoo.com

\section{References}

1 Wakayama S, Hikichi T, Suetsugu R, et al. Stem Cells 2007; 25:986993.

2 Wakayama T, Hayashi Y, Ogura A. J Reprod Fertil 1997; 110:263266.

3 Wakayama T, Yanagimachi R. Biol Reprod 1998; 59:100-104.

4 Wang T, Sha H, Ji D, et al. Cell 2014; 157:1591-1604.

5 Wolf DP, Mitalipov N, Mitalipov S. Trends Mol Med 2015; 21:68-76.

6 Ma H, O'Neil RC, Marti Gutierrez N, et al. Cell Stem Cell 2017; 20:112-119.

7 Tachibana M, Sparman M, Mitalipov S. Nat Protoc 2010; 5:11381147.

8 Wu K, Chen T, Huang S, et al. Cell Res 2017; 27:834-837.

9 Harton GL, Munne S, Surrey M, et al. Fertil Steril 2013; 100:16951703.

10 Alfarawati S, Fragouli E, Colls P, et al. Fertil Steril 2011; 95:520524.

11 Pinheiro LB, Coleman VA, Hindson CM, et al. Anal Chem 2012; 84:1003-1011.

12 Dalton CM, Carroll J. J Cell Sci 2013; 126:2955-2964.

(Supplementary information is linked to the online version of the paper on the Cell Research website.) 